\title{
Surface tension and dynamics of fingering patterns
}

\author{
F. X. Magdaleno and J. Casademunt \\ Departament d'Estructura i Constituents de la Matèria, Universitat de Barcelona, Avenida Diagonal, 647, E-08028 Barcelona, Spain
} (Received 22 December 1997)

\begin{abstract}
We study the minimal class of exact solutions of the Saffman-Taylor problem with zero surface tension, which contains the physical fixed points of the regularized (nonzero surface tension) problem. New fixed points are found and the basin of attraction of the Saffman-Taylor finger is determined within that class. Specific features of the physics of finger competition are identified and quantitatively defined, which are absent in the zero surface tension case. This has dramatic consequences for the long-time asymptotics, revealing a fundamental role of surface tension in the dynamics of the problem. A multifinger extension of microscopic solvability theory is proposed to elucidate the interplay between finger widths, screening and surface tension. [S1063-651X(98)51204-4]

PACS number(s): 47.54.+r, 47.20.Hw, 47.20.Ky, 47.20.Ma
\end{abstract}

The displacement of a viscous fluid by a nonviscous one within the gap of a Hele-Shaw cell [1] has been one of the most studied problems in interfacial pattern formation for several decades [2]. The relative simplicity of the problem has made possible an analytical understanding of the subtle role of surface tension $\sigma$ in the selection of the SaffmanTaylor finger [3], as a prototype of the so-called microscopic solvability (MS) scenario of pattern selection [2]. More recently, the increasingly interesting and controversial issue of the role of surface tension in the dynamics of fingering patterns has been raised.

It is known that the zero surface tension Saffman-Taylor (ST) problem is ill-posed as an initial value problem and is plagued of finite-time singularities $[4,5]$. Studies of Laplacian growth with zero surface tension, however, have proven insightful, for instance, in cases with needlelike growth [6]. In the case of smooth interfaces which concerns us here, a rich variety of physically relevant morphologies has been found among solutions of the $\sigma=0$ problem which remain smooth all the time (free of finite-time singularities) $[4,7,8]$. Given the difficulty to get analytical information from the $\sigma \neq 0$ problem, this has raised the question of what part of the physics of fingering dynamics, if any, is captured by those solutions. Within this spirit, Dai, Kadanoff, and Zhou explored via numerical simulation the qualitative differences of distinct classes of initial conditions [9]. For the so-called polelike class and for finite time, the problem was concluded to be qualitatively similar with and without surface tension. More recently, Siegel and Tanveer [10] have shown that the regularized problem (i.e., vanishingly small $\sigma$ ) may differ significantly from the idealized problem $(\sigma=0)$ in order one time, and therefore, smooth time evolving solutions of the $\sigma=0$ problem do not coincide, in general, with the limit of solutions of the regularized problem. Since evidence of this is found only for the particular case of single-finger configurations, the conclusion may not apply to other situations [11]. Furthermore, from a less stringent standpoint one could seek for situations where the evolution with and without surface tension are at least qualitatively equivalent, in the sense that a (small) quantitative difference between the two remains bounded for all time [12]. Further physical insight is thus still necessary to clarify the phenomenology which may be appropriately captured by the idealized problem, particularly concerning the long-time asymptotics.

From a different perspective, the MS scenario itself has been questioned recently by results of Ref. [13], where it is claimed that in a wide class of nonsingular exact solutions, the $\sigma=0$ dynamics leads naturally to the solution predicted by selection theory, without invoking surface tension to explain selection. This seems to support the claim, in clear contradiction with Ref. [10], that surface tension is unessential to the dynamics. In this paper we sustain the opposite conclusion $[14,15]$.

Our approach here consists of identifying specific dynamical features that can be viewed as essential to the process of finger competition from a physical standpoint, and check them in exactly solvable zero surface tension cases. This will allow for a precise diagnosis on the physical content of the idealized $\sigma=0$ problem.

The evolution equation for the time-dependent conformal mapping $f(w, t)$ of the interior of the unit circle in the complex plane $w$ into the region occupied by the viscous fluid in the physical plane $z=x+i y$, in the case of zero surface tension and in the comoving frame (the frame moving with the mean interface velocity) can be written as

$$
\operatorname{Re}\left\{w \partial_{w} f(w, t)\left[1+\partial_{t} f^{*}(w, t)\right]\right\}=-1 .
$$

This is an alternative form to the equation of motion described for instance in Refs. [1] or [7]. An infinite channel of width $2 \pi$ is considered along the $x$ axis, and periodic boundary conditions are assumed in the $y$ direction.

The basic idea is to find a simple, low-dimensional nonsingular class of solutions of Eq. (1) which contains the physical fixed points of the regularized problem, and compare the phase space flow topology in both cases. The key point is that we do not need to know the exact phase space trajectories of the regularized problem, which are particularly difficult to obtain even numerically for long times, but only the phase space flow topology. The latter can indeed be inferred unambiguously from existing empirical evidence both experimentally and simulationally. For finite surface tension, a two-finger configuration subspace, for instance, must contain three fixed points, namely the (unstable) planar interface 
(PI), the (stable) ST single finger (1ST), and a saddle fixed point corresponding to the degenerate double ST finger (2ST). For finite $\sigma$ (and in the high viscosity contrast limit [16]), the 1ST fixed point is known to be the universal attractor of this problem so all trajectories start at PI and end up at $1 \mathrm{ST}$. The $2 \mathrm{ST}$ fixed point will have a lower dimension attracting manifold conected to PI, and will presumably govern the dynamics of finger competition.

A class of solutions with $\sigma=0$ which contains planar, one-finger, and two-finger fixed points is of the form

$$
\begin{aligned}
f(w, t)= & -\ln w+d(t)+(1-\lambda)\{\ln [1-\alpha(t) w] \\
& \left.+\ln \left[1+\alpha^{*}(t) w\right]\right\},
\end{aligned}
$$

where $\alpha(t)=\alpha^{\prime}(t)+i \alpha^{\prime \prime}(t)$. This corresponds generically to two unequal fingers. The interface shape has two symmetry axes along the fingers, separated a distance $\pi$. Such symmetry simplifies the analysis but does not affect the competition of fingers in any fundamental way [16].

The case $\alpha^{\prime}(t)=0$ corresponds to a single finger with the asymptotic ST shape for $\alpha^{\prime \prime} \rightarrow 1$. The case $\alpha^{\prime \prime}(t)=0$ corresponds to two identical fingers which tend to a doubly degenerate ST solution as $\alpha^{\prime} \rightarrow 1$. For $|\alpha(t)| \ll 1$ the ansatz (2) describes sinusoidal perturbations of a planar interface [17]. $\lambda$ is a constant of motion and takes real values in the interval $[0,1]$. For well developed fingers $\lambda$ is the total filling fraction of the channel occupied by the invading fingers.

For $\sigma=0$ this ansatz is exactly solvable in the sense that if we insert Eq. (2) into Eq. (1) we obtain a closed set of ordinary differential equations for the parameters $\alpha^{\prime}, \alpha^{\prime \prime}$, and $d$. According to Ref. [7] this case is free of finite-time singularities. The parameter $d$ accounts for a global displacement and is irrelevant for the present discussion.

For the sake of discussion and visualization, we find convenient to parametrize the phase space in terms of the variables $u=1-\alpha^{\prime \prime 2}$ and $r=\left(\alpha^{\prime 2}+\alpha^{\prime \prime 2}-1\right) /\left(\alpha^{\prime \prime 2}-1\right)$. Thus, the phase space is the cube $[0,1] \times[0,1] \times[0,1]$ in the $(u, r, \lambda)$ space. In these variables, the time evolution is given by the equations

$$
\begin{gathered}
\dot{u}=2 r u(1-u) \frac{3 r-4-g r(1-r u)}{1+g T_{g}(u, r)}, \\
\dot{r}=2 r(1-r) \frac{3 r-2(1+r u)+g(1-r u)(2-r)}{1+g T_{g}(u, r)},
\end{gathered}
$$

where

$$
\begin{aligned}
T_{g}(u, r)= & (1-g)[2 r+g(2 r-1)]-\frac{1}{2}(1-g)^{2} r u \\
& -g u r^{2}[1+g(r u-3)]
\end{aligned}
$$

and where $g=1-2 \lambda=$ const.

In order to compare with the physical case of $\sigma \neq 0$ we introduce the following construction. Consider a onedimensional set of initial conditions $(t=0)$ of the form Eq. (2) surrounding the planar interface (PI) fixed point $u=1, r$ $=1$, for a fixed $\lambda$. We take them infinitesimally close to PI in such a way that the interface is in the linear regime [17]. The time evolution from $t=-\infty$ to $t=\infty$ of this set spans a com-

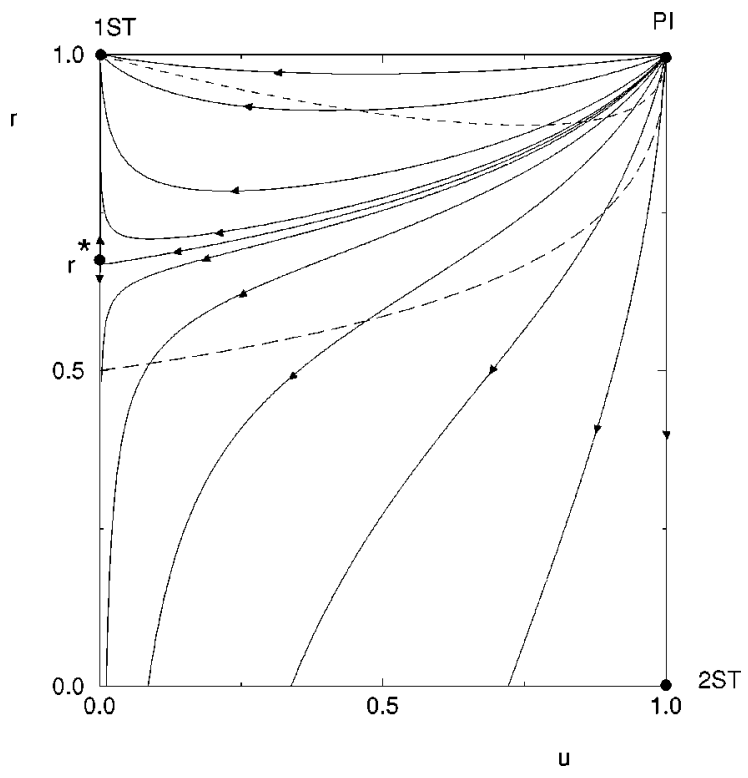

FIG. 1. Phase space flow of the dynamical system defined by Eqs. (3) $-(5)$ for $\lambda=1 / 2$. See explanation in text.

pact two-dimensional phase space $\left(u^{\prime}, r^{\prime}\right)$ embedded in the infinite dimensional space of interface configurations. We may define the space $\left(u^{\prime}, r^{\prime}\right)_{0}$ as the limiting case of $\sigma \rightarrow 0$ (taken after the limits $t \rightarrow \pm \infty$ ). Since in the linear regime the regularized problem for vanishingly small $\sigma$ converges regularly to the $\sigma=0$ solution, the manifolds $(u, r)$ and $\left(u^{\prime}, r^{\prime}\right)$ must be tangent at the PI fixed point, $u=1$, $r=1$ (see Fig. 1). According to selection theory, for $\sigma \rightarrow 0$, the 1ST and 2ST fixed points must approach the corresponding single-finger and double-finger fixed points of Eqs. (3)(5) with $\lambda=1 / 2$, which occur at $u=0, r=1$ and $u=1, r$ $=0$ respectively. Furthermore, we expect that the limiting case $\left(u^{\prime}, r^{\prime}\right)_{0}$ and the space $(u, r)$ with $\lambda=1 / 2$ must intersect not only at PI, $1 \mathrm{ST}$, and $2 \mathrm{ST}$, but they must have in common the two lines $r=1$ and $u=1$.

Following the topological approach of Ref. [16], it is useful to consider the stream function $\psi$, defined as the imaginary part of the complex potential $\Phi(w, t)=-f(w, t)$ $-\ln w$, in the comoving frame. Along the interface, $\psi$ is then a periodic function which provides a natural definition of individual aerial growth rate of fingers, which we call $\Delta \psi_{L}$ and $\Delta \psi_{S}$ for the longer and shorter fingers respectively. In this simple case these are given as maximum-to-minimum differences of the stream function extrema along the interface. $\psi$ may have only one maximum even for two-finger configurations, in which case we take $\Delta \psi_{S}=0$ and qualify the finger as " "nongrowing." In our case, and for finite width fingers, $\Delta \psi>0$ (growing) and $\Delta \psi=0$ (nongrowing) correspond respectively to positive and negative tip velocities relative to the mean interface position.

The physical scenario of finger competition which we want to test, extracted from experiments and simulations [16] can be briefly described as follows. In the linear and early nonlinear regimes two different fingers grow with both $\Delta \psi_{L}(t)$ and $\Delta \psi_{S}(t)$ increasing with time. When the fingers are well developed and the "growth" function $G(t)$ $=\left[\Delta \psi_{L}(t)+\Delta \psi_{S}(t)\right] /(1-\lambda) \quad\left[\right.$ with $\quad 1-\lambda=\Delta \psi_{L}(\infty)$ $\left.+\Delta \psi_{S}(\infty)\right]$ is of order 1 , the competition takes over. This is 
signaled by an enhanced growth of the "competition" function $C(t)=\left(\Delta \psi_{L}(t)-\Delta \psi_{S}(t)\right) /\left(\Delta \psi_{L}(t)+\Delta \psi_{S}(t)\right)$ as $\Delta \psi_{L}$ starts to increase at the expense of $\Delta \psi_{S}$. Existence of competition can thus be identified with $\Delta \psi_{S}$ decreasing with time. The competition may be termed "successful" when $\Delta \psi_{S} \rightarrow 0$ asymptotically $[C(\infty)=1]$, that is when a "growing" finger is turned into "nongrowing" due to the presence of another finger. As discussed in Ref. [16], this dynamical elimination of the small finger is associated to topology changes in the physical velocity field, which occur via the crossing of topological defects through the interface.

We now discuss the zero surface tension dynamics of our ansatz (2), by analyzing the phase portrait of the dynamical system defined by Eqs. (3)-(5). This is plotted in Fig. 1 for $\lambda=1 / 2$. In this case Eqs. (3) $-(5)$ can be integrated analytically. Dynamical trajectories are of the form

$$
\frac{2 u-3 r u+r^{2} u^{2}}{\sqrt{u(1-r)(1-r u)}}=\mathrm{const}
$$

and are plotted as solid lines with arrows. The dashed lines are $\lambda$-independent boundaries. The short-dashed line separates the one-finger (above) and the two-finger (below) regions. The long-dashed one is the defect boundary separating the no-defect (above) and the 1-defect (below) regions, with $\Delta \psi_{S}=0$ and $\Delta \psi_{S}>0$ respectively.

Our central result is that the topological structure of the phase portrait for $\sigma=0$ (Fig. 1) is radically different from that of finite surface tension. Its most salient feature is the fact that the ST single-finger solution has a limited basin of attraction. Part of the flow evolves towards a continuum of (attractive) fixed points $r=0$. The separatrix of the two regions is a critical trajectory ending in a new (saddle) fixed point located at $u^{*}=0$ and $r^{*}=2 \lambda /(1+\lambda)$.

For arbitrary $\lambda$, the line $r=0$ is a continuum of stationary solutions with coexisting unequal fingers (different widths $\lambda_{1}, \lambda_{2}$ with $\lambda_{1}+\lambda_{2}=\lambda$ ) advancing with the same velocity and with tip positions separated in the $x$ direction by a finite distance $\Delta=(1-\lambda) \log [(1+\sqrt{1-u}) /(1-\sqrt{1-u})]$. Solutions of this type have been reported previously $[7,8]$. We would like to call the attention upon the fact that the screening of the Laplacian field, as the mechanism usually invoked to explain competition, applies actually to the aerial growth of the small finger $\Delta \psi_{S}$, which is reduced indeed by the presence of the longer neighboring finger, but not to the velocity, which may be in fact the same. Concepts such as screening length and time in competing fingers such as used in [6], are only well defined after and additional constraint fixing the relative finger widths is supplied.

The fixed point $\left(u^{*}, r^{*}\right)$ corresponds to a new type of asymptotic stationary solution of the $\sigma=0 \mathrm{ST}$ problem. It consists of two fingers with unequal positive velocities. The length ratio of the fingers satisfies [15] $\lim _{t \rightarrow \infty} L_{S} / L_{L}=1 / 3$ independently of $\lambda$. For the $r=0$ solutions we have $\lim _{t \rightarrow \infty} L_{S} / L_{L}=1$ while, for the 1 ST fixed point when approached from the two-finger region we have $\lim _{t \rightarrow \infty} L_{S} / L_{L}$ $=0$. In the latter case, the residual nongrowing finger which subsists is reminiscent of the "frozen" fingers observed in real experiments.
Our basic point is that, according to the above discussion, the possibility of successful competition is associated to the fact that dynamical trajectories cross the defect boundary from below (annihilation of topological defects [16]). In Fig. 1 we see that, for $\lambda=1 / 2$, there is no successful competition whatsoever since the critical trajectory is located above the defect boundary [18].

The cases of $\lambda \neq 1 / 2$ and $\sigma=0$ are not directly relevant to the viscous fingering problem, but may be relevant to other generic situations of Laplacian growth in the spirit of Refs. [6], and will be discussed in detail elsewhere [15]. Here we will just remark that $r^{*}(\lambda)$ is monotonically increasing, between $r^{*}(0)=0$ and $r^{*}(1)=1$. Therefore, the basin of attraction of the single finger solution is larger for narrower fingers. Furthermore, since the defect boundary is independent of $\lambda$, there exists a critical $\lambda_{c}=1 / 3$ for which $r^{*}$ crosses the defect boundary. This implies that, for $\lambda<1 / 3$, there are dynamical trajectories which cross the defect boundary from below, and therefore the competition is then successful for some finite region of phase space.

In summary, from the analysis of the $\sigma=0$ dynamics of the class Eq. (2) we conclude that (i) only a small $\lambda$ dependent part of phase space behaves qualitatively as the $\sigma \neq 0$ problem, leading to a ST single finger (with maybe a residual non-growing finger); (ii) dynamical elimination of growing fingers does not occur for finger widths relevant to the problem of viscous fingering $(\lambda=1 / 2)$; (iii) the picture of competition based solely on Laplacian screening is insufficient, since relative widths of fingers and not only relative tip positions come into play.

In order for the "screening" picture to be valid, an additional dynamical constraint is required to force the finger widths to be equal. In growth processes based on aggregation of particles, the finger width may be fixed by particle size (set to zero in Refs. [6]). In the problem of viscous fingering such constraint is supplied precisely by surface tension. This suggests that an extension of MS, which is essentially a static theory, to multifinger configurations, may shed new light on the dynamics of the problem.

The generalized multifinger MS scenario can be sketched as follows. For two-finger configurations, there exists a two parameter continuum family of steady state solutions which we can parametrize by $\lambda=\lambda_{1}+\lambda_{2}$ and $p=\lambda_{1} /\left(\lambda_{1}+\lambda_{2}\right)$. The cases $p=0,1$ correspond trivially to the single-finger case. The case $p=1 / 2$ (two identical fingers) is also reducible to single-finger MS in a channel of half width. Most interestingly, one can show [19] that, for nonzero surface tension, nontrivial stationary solutions with unequal fingers $(p \neq 1 / 2)$ exist. In this case, surface tension selects an infinite set of values of $\lambda$ which differs from the single-finger case, but which scale also as $(\lambda-1 / 2) \sim \sigma^{2 / 3}$. Furthermore, for any given $\lambda$ of the above discrete set, there exists another countably infinite set of possible values of $p$ with $(p-1 / 2) \sim$ $\pm \sigma^{1 / 3}[19]$. We plan to present this new set of fixed points of the problem with surface tension and its physical relevance elsewhere [19]. It is reasonable to expect that all two-finger solutions except the $p=1 / 2, \lambda=\lambda_{m}(\sigma)$ (with $\lambda_{m}$ the minimum value of $\lambda$ from MS) lie outside the space $\left(u^{\prime}, r^{\prime}\right)$. Despite the fact of being globally unstable, the $p=1 / 2, \lambda$ $=\lambda_{m}$ fixed point is then the physically relevant one to describe finger competition since it has an attracting manifold 
which includes the PI fixed point (linear regime). The process of finger competition can thus be pictured as follows. From the linear instability a given number of fingers emerge. As far as this early stage is dominated by the most linearly unstable mode (in the limit of weak white noise on PI, the emerging configuration will indeed be nearly periodic), the interface is relatively close to the attracting manifold of the $n \mathrm{ST}$ fixed point $\left[n\right.$ equal fingers with $\left.\lambda_{i}=\lambda_{m}(\sigma) / n\right]$. The fingers tend thus to adopt the same fingertip curvature and select their widths at early stages of the nonlinear regime according to single-finger MS theory. The $n \mathrm{ST}$ fixed will then govern the process of competition in the sense that the path connecting typical initial configurations with the single finger attractor, must necessarily pass near that saddle point. The phenomenon of competition is then viewed as the crossover to the unstable directions of the $n \mathrm{ST}, \lambda=\lambda_{m}$ fixed point. Such crossover is what is missed in the $\sigma=0$ problem, since the unstable direction of the 2ST fixed point becomes infinitelly marginal (a line of fixed points) in that limit. In the terminology of dynamical systems, this reflects the fact that Eqs. (3)-(5) are structurally unstable [20]. We thus conclude that surface tension plays a fundamental role in the dynamics of finger competition and that, for the long time asymptotics, it can only be treated as a "regular' perturbation in a very limited region of phase space which excludes multifinger configurations.

We are indebted to David Jasnow for stimulating discussions. We acknowledge financial support from the Dirección General de Enseñanza Superior (Spain), Project No. PB961001-C02-02, and the European Commission Project No. ERB FMRX-CT96-0085. F.X.M. also acknowledges financial support from the Comissionat per a Universitats i Recerca (Generalitat de Catalunya).
[1] D. Bensimon, L. Kadanoff, S. Liang, B. I. Shraiman, and C. Tang, Rev. Mod. Phys. 58, 977 (1986).

[2] P. Pelcé, in Perspectives in Physics (Academic Press, New York, 1988); D. A. Kessler, J. Koplik, and H. Levine, Adv. Phys. 35, 255 (1988).

[3] B. I. Shraiman, Phys. Rev. Lett. 56, 2028 (1986); D. C. Hong and J. S. Langer, ibid. 56, 2032 (1986); R. Combescot, T. Dombre, V. Hakim, Y. Pomeau, and A. Pumir, ibid. 56, 2036 (1986).

[4] S. D. Howison, J. Fluid Mech. 167, 439 (1986); S. D. Howison, J. R. Ockendon, and A. A. Lacey, Q. J. Mech. Appl. Math. 38, 343 (1985).

[5] S. Tanveer, Philos. Trans. R. Soc. London, Ser. A 343, 155 (1993).

[6] B. Derrida and V. Hakim, Phys. Rev. A 45, 8759 (1992); J. Krug, K. Kassner, P. Meakin, and F. Family, Europhys. Lett. 24, 527 (1993).

[7] S. P. Dawson and M. Mineev-Weinstein, Physica D 73, 373 (1994).

[8] G. Baker, M. Fisher, and S. Tanveer, J. Comput. Phys. 120, 348 (1994).

[9] W. Dai, L. P. Kadanoff, and S. Zhou, Phys. Rev. A 43, 6672 (1991)

[10] M. Siegel and S. Tanveer, Phys. Rev. Lett. 76, 419 (1996).
[11] For instance, a sequence of solutions of $\sigma \neq 0$ can be found which converges uniformly for all time to the $\lambda=1 / 2$ timedependent solution with $\sigma=0$.

[12] For instance, single-finger evolutions with $\lambda>1 / 2$.

[13] M. Mineev-Weinstein, Phys. Rev. Lett. 80, 2113 (1998).

[14] We do not question the validity of the analytical results of Ref. [13] but the conclusions drawn concerning the dynamical role of surface tension. In particular, we point out that any $\lambda$ $\neq 1 / 2$ may be dynamically selected within the appropriate subspace. Furthermore, the dynamics leading to $\lambda=1 / 2$ need not be close to the physical one. For more details, see Ref. [15].

[15] F. X. Magdaleno and J. Casademunt (unpublished).

[16] J. Casademunt and D. Jasnow, Phys. Rev. Lett. 67, 3677 (1991); J. Casademunt and D. Jasnow, Physica D 79, 387 (1994).

[17] Two modes $k=1,2$ with amplitudes $\left|\epsilon_{1}\right| \sim\left|\epsilon_{2}\right| \ll 1$ satisfy $\epsilon_{1}$ $\sim \alpha^{\prime \prime}$ and $\epsilon_{2} \sim \alpha^{\prime 2}$.

[18] Strictly speaking, there may be a certain amount of competition in the sense of a decrease of $\Delta \psi_{S}(t)$ but quantitatively very small.

[19] F. X. Magdaleno and J. Casademunt (unpublished).

[20] See Peixoto's theorem in J. Guckenheimer and P. Holmes, Nonlinear Oscillations, Dynamical Systems and Bifurcations of Vector Fields (Springer-Verlag, Berlin, 1983). 